\title{
RESPONSE BOUNDS FOR HYSTERETIC SECOND ORDER SYSTEMS*
}

BY

N. MOSTAGHEL

University of Utah

The behavior of many engineering systems is governed by the second order differential equation

$$
\ddot{U}(t)+F[U(t)]=\ddot{G}(t),
$$

where $\ddot{G}(t)$ is a specified oscillatory function of time $t$, a dot denotes differentiation with respect to $t, F(U)$ is a nonlinear restoring function representing the system hysteresis, as shown in Fig. 1, and $U(0)=\dot{U}(0)=0$. In a recent paper [1], it has been shown that

$$
f=F(u) \leqslant(1 / \alpha) \ddot{g},
$$

where $f=\sup |F(U)|, u=\sup |U(t)|, \ddot{g}=\sup |\ddot{G}(t)|$, and $0<\alpha \leqslant 1$ is given by

$$
\alpha=\frac{A}{4 f u},
$$

where $A$ is the area of the hysteresis loop.

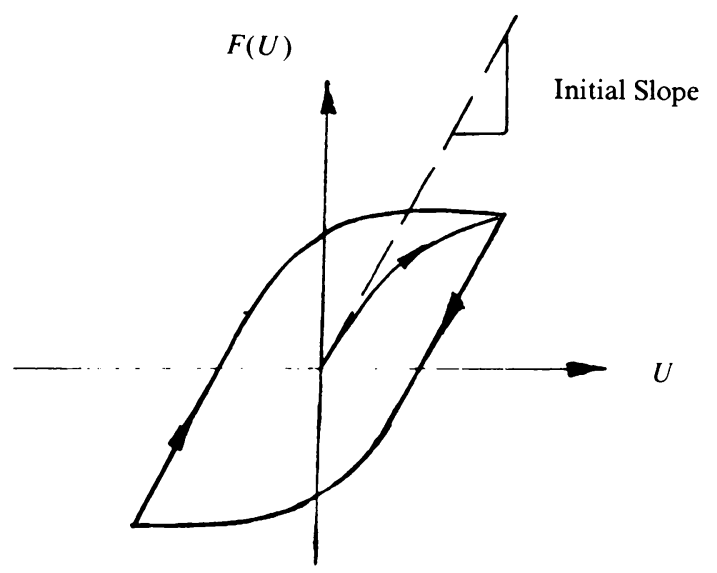

FIG. 1. Representation of $F(U)$

${ }^{*}$ Received December 16, 1985. 
The quality of the upper bound on $U(t)$, as given by inequality (2), deteriorates with the reduction in the initial slope of $F(U)$. That is, the estimate provided by inequality (2) is good for stiff systems. For soft systems (i.e., systems for which the initial slope of $F(U)$ is small), this estimate may be much higher than the actual values of $U(t)$.

To find a bound on $U(t)$ suitable for soft systems, both sides of Eq. (1) are multiplied by $\dot{U}(t)$, and the resulting expression is integrated over the interval $\left[t_{i}, t_{i+1}\right]$ to yield

$$
\int_{t_{i}}^{t_{i+1}} F(U) \dot{U}(t) d t=\int_{t_{i}}^{t_{i+1}} \ddot{G}(t) \dot{U}(t) d t,
$$

where $t_{i}$ and $t_{i+1}$ are two consecutive times of zero crossing of $\dot{U}(t)$. If both sides of Eq. (1) are multiplied by $\dot{G}(t)$ and the resulting expression is integrated over the same interval $\left[t_{i}, t_{i+1}\right]$, it yields

$$
\left.\int_{t_{i}}^{t_{i+1}} \ddot{U}(t) \dot{G}(t) d t+\int_{t_{i}}^{t_{i+1}} F(U) \dot{G}(t) d t=\frac{1}{2} \dot{G}^{2}(t)\right]_{t_{i}}^{t_{i+1}} .
$$

Since $t_{i}$ and $t_{i+1}$ are the times of zero crossing for $\dot{U}(t)$, integration by parts of the first term on the left-hand side of the above expression yields

$$
\int_{t_{i}}^{t_{i+1}} \ddot{U}(t) \dot{G}(t) d t=-\int_{t_{i}}^{t_{i+1}} \dot{U}(t) \ddot{G}(t) d t .
$$

Comparing Eqs. (4) and (6), one obtains

$$
\int_{t_{i}}^{t_{i+1}} \ddot{U}(t) \dot{G}(t) d t=-\int_{t_{i}}^{t_{i+1}} F(U) \dot{U}(t) d t .
$$

Substitution from Eq. (7) into Eq. (5) results in

$$
\int_{t_{i}}^{t_{i+1}} F(U) \dot{U}(t) d t+\frac{1}{2} \dot{G}^{2}\left(t_{i+1}\right)=\int_{t_{i}}^{t_{i+1}} F(U) \dot{G}(t) d t+\frac{1}{2} \dot{G}^{2}\left(t_{i}\right) .
$$

Since $F(U)$ has at most one zero crossing in the time interval $\left[t_{i}, t_{i+1}\right]$, then

$$
\left|\int_{t_{i}}^{t_{i+1}} F(U) \dot{G}(t) d t\right|=\left|\int_{G\left(t_{1}\right)}^{G\left(t_{t+1}\right)} F(U) d G\right| \leqslant 2 f g,
$$

where $g=\sup |G(t)|$. Also,

$$
\left|\int_{t_{i}}^{t_{i+1}} F(U) \dot{U}(t) d t\right|=\left|\int_{U\left(t_{i}\right)}^{U\left(t_{i+1}\right)} F(U) d U\right|=\alpha(2 f u),
$$

where $\alpha$ is the reduction factor which makes the equality satisfied, and its value is given by relation (3). Comparisons of equalities (8) and (10) and inequality (9) yield

$$
\alpha f u \leqslant f g+\left(\frac{1}{4}\right) \dot{g}^{2},
$$

where $\dot{g}=\sup |\dot{G}(t)|$. The simultaneous occurrence of $f$ and $u$ is an implicit assumption in both inequalities (2) and (11). Equivalently, inequality (11) can be written as

$$
\alpha u F(u) \leqslant g F(u)+\left(\frac{1}{4}\right) \dot{g}^{2} .
$$


This inequality yields an upper bound on $u$ with $\alpha$ as a parameter suitable for soft systems.

Acknowledgment. The support of the National Science Foundation under Grant CEE8414504 is gratefully acknowledged.

\section{REFERENCES}

[1] N. Mostaghel, A response bound for hysteretic second order systems, Quart. Appl. Math. XLIII, 199-200 (1985) 